\title{
ENGINEERING JOURIIAL
}

\section{Reviere}

\section{Howling and Entrainment in Hearing Aids: A Review}

\author{
Muhammad Tahir Akhtar ${ }^{1, a, *}$ and Akinori Nishihara ${ }^{2, b}$ \\ 1 Department of Electrical Engineering, COMSATS Institute of Information Technology, Park Road, \\ Chak Shahzad, Islamabad 44000, Pakistan \\ 2 Human Assets Promotion Program for Innovative Education and Research (HAPPIER), Tokyo \\ Institute of Technology, Ookayama, Meguro-ku, Tokyo, 152-8552 Japan

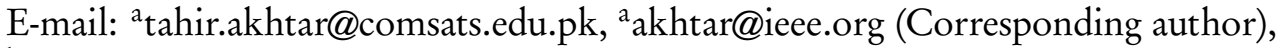 \\ baki@cradle.titech.ac.jp
}

\begin{abstract}
This review focuses on howling and entrainment artifacts in digital hearing aids. The howling may occur (especially at high gains), essentially due to the close proximity of the input microphone and the output loudspeaker. The entrainment, on the other hand, occurs when the input to the hearing aids is periodic, for example, music signals or alarm signals with strong tonal characteristics. We give details on methods for howling avoidance, which are mainly based on adaptive filtering-based acoustic feedback cancellation. We also give an overview of many recent works on entrainment in hearing aids. Finally, we remark that efficient acoustic feedback cancellation scheme which can avoid howling, can also well manage the entrainment artifact.
\end{abstract}

Keywords: Hearing aids, adaptive filtering, NLMS algorithm, acoustic feedback, howling, entrainment.

ENGINEERING JOURNAL Volume 20 Issue 5

Received 23 September 2016

Accepted 23 September 2016

Published 25 November 2016

Online at http://www.engj.org/

DOI:10.4186/ej.2016.20.5.5 
Notations: A discrete-time (DT) signal is represented as $x(n) \stackrel{z}{\longleftrightarrow} X(z)$ where $x(n)$ denotes the time-domain representation and $X(z)$ being its corresponding $z$-transform. For a DT system being represented as $h(n) \stackrel{z}{\longleftrightarrow} H(z), h(n)$ denotes the impulse response characteristics and $H(z)$ being the corresponding transfer function. Without loss of generality all DT systems; acoustic path, hearing aid processing unit, adaptive filter, etc.; are assumed to be modeled as finite impulse response (FIR) filters. Thus for a DT system represented as $h(n) \stackrel{z}{\longleftrightarrow} H(z)$, the corresponding impulse response coefficient vector can be written as $\boldsymbol{h}(n)=\left[h_{0}(n), h_{1}(n), \cdots, h_{M-1}(n)\right]^{T}$, where ${ }^{T}$ denotes transpose operation, $M$ is the tap-weight length, and where the DT index ' $n$ ' may be dropped for time-invariant systems.

\section{Introduction}

Hearing loss involves shift in auditory threshold, changes in the system input/output gain behavior, and the loss of frequency and temporal resolution. The development of signal processing to compensate for these changes in the impaired ear presents a significant engineering challenge. As shown in a simplified block diagram in Fig. 1(a), a hearing aid typically comprises a microphone to pick-up the input signal $s(n) \stackrel{z}{\longleftrightarrow} S(z)$, a signal processing block $G(z)$ essentially to perform the amplification and a receiver (loudspeaker). Here $G(z)$ represents the forward path of the hearing aid and comprises all signal processing for noise reduction and signal amplification and may comprise sub-band processing in accordance with the hearing loss characteristics of the user. Since hearing aid is a small device, the microphone and the receiver are located in a close proximity. Furthermore, the hearing aid cannot be tightly fitted as it would cause discomfort to the hearing aid user. This creates a feedback (leakage) path (shown as $F(z)$ in Fig. 1(a).) between the receiver and the microphone. The receiver signal $y(n) \stackrel{z}{\longleftrightarrow} Y(z)$ is not only propagated to user ear, but is also fed back to the input microphone which creates a closed-loop system. This is called acoustic feedback in hearing aids. In Fig. 1(a), the closed loop transfer function between the received signal $y(n) \stackrel{z}{\longleftrightarrow} Y(z)$ and the input signal $s(n) \stackrel{z}{\longleftrightarrow} S(z)$ is given as:

$$
H(z)=\frac{Y(z)}{S(z)}=\frac{G(z)}{1-G(z) F(z)} .
$$

Equation (1) shows that due to acoustic feedback, the hearing aid may become unstable if $G(z)$ is large enough so that $G(z) F(z)=1$ at some frequency. Acoustic feedback is a major problem in hearing aids, limiting the maximum gain available to user, and making hearing aids oscillate at higher gain thus producing annoying sounds of whistling, screeching or so-called the howling effect. Thus reduction of acoustic feedback is very important in the context of hearing aids.

A literature review shows that a number of approaches have been proposed to solve the problem of acoustic feedback and to allow hearing aid users to obtain higher stable gains. See for example [1]-[5] for some classical works, and for a detailed review on various proposals for AFC in hearing aids, reader is referred to [6] and [7]. Noting that the acoustic feedback path between the receiver (loudspeaker) and the microphone may vary suddenly and drastically, for example, when user brings mobile phone near his/her ear [8]. Therefore, in order to deal with the time-varying nature of the acoustic feedback path, the adaptive feedback cancellation (AFC) techniques have become an integral part of the modern hearing aids. As shown in Fig. 1(b), AFC is achieved by an adaptive filter $W(z)$ to model the acoustic feedback path $F(z)$. The most famous adaptive algorithm employed for adaptation of the AFC filter $W(z)$ is the normalized least mean square (NLMS) algorithm [9], which is due to its simplicity, robustness and ease of implementation. It is evident from Fig. 1(b) that the received signal $y(n) \stackrel{z}{\longleftrightarrow} Y(z)$, and the microphone signal $x(n)=s(n)+y_{f}(n) \stackrel{z}{\longleftrightarrow} X(z)=S(z)+Y_{f}(z)$, act as the input signal and desired response, respectively, for $W(z)$. These two signals are strongly correlated with each other, and this results in a biased convergence of $W(z)$ and, hence, a non-optimal cancellation of acoustic feedback. Developing efficient adaptive filtering methods has been focus of many researchers in the past few decades. The first objective of the present review is to highlight key works in this area.

The second goal of this paper is to present a detailed review of state-of-art methods for entrainment containment in hearing aids. The "entrainment" artifact is encountered when the input signal has strong 


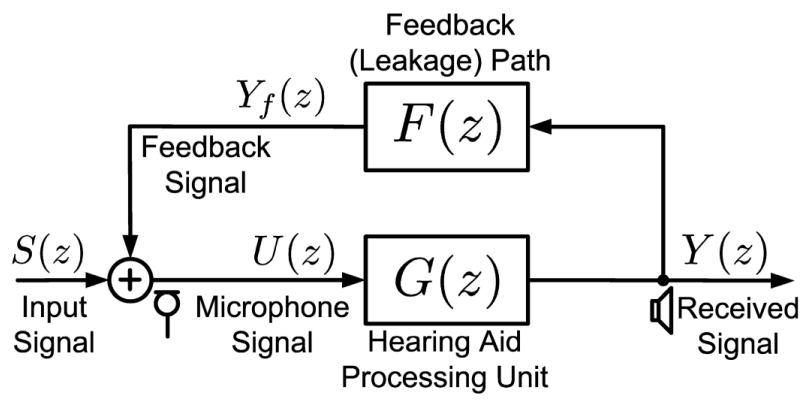

(a)

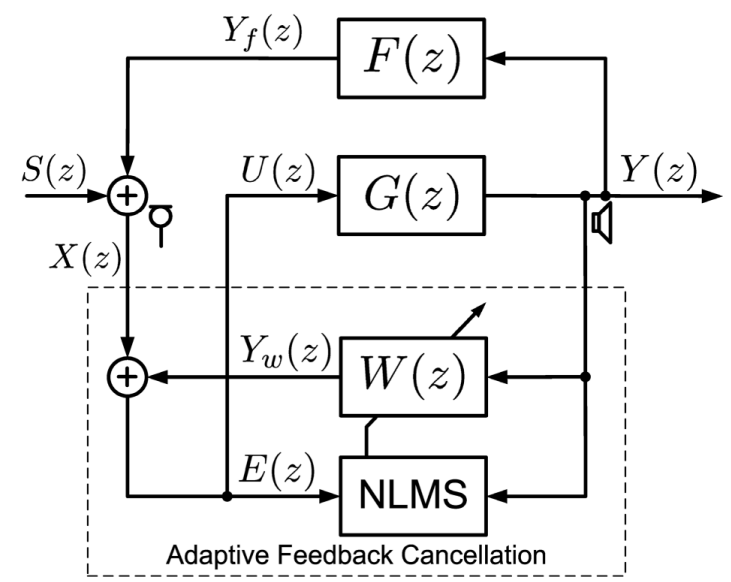

(b)

Fig. 1. (a) A simplified block diagram of hearing aid under acoustic feedback. (b) A block diagram of NLMS algorithm-based conventional method for adaptive feedback cancellation (AFC).

tonal characteristics, which is the case for languages like Mandarin/Cantonese, music signals, alarms, ringing bells, etc. We attempt to explain entrainment first from physics of relation and interaction between two independent rhythmic processes, and then describe recent works on entrainment in hearing aids. Finally, we conclude this paper by giving a few remarks.

\section{Adaptive Feedback Cancellation}

In the conventional approach for AFC in hearing aids (shown in Fig. 1(b) in its simplest form), the coefficient of $W(z)$ are updated using NLMS algorithm as:

$$
\boldsymbol{w}(n+1)=\boldsymbol{w}(n)+\frac{\mu}{\boldsymbol{y}^{T}(n) \boldsymbol{y}(n)+\delta} e(n) \boldsymbol{y}(n),
$$

where $\mu$ is the step-size parameter, $\delta$ is a small positive constant to avoid division by zero, $\boldsymbol{w}(n)=$ $\left[w_{0}(n), w_{1}(n), \cdots, w_{M-1}(n)\right]^{T}$ is the coefficient vector for $W(z), \boldsymbol{y}(n)=[y(n), y(n-1), \cdots, y(n-$ $M+1)]$ is the received signal vector comprising $M$-recent samples of the received signal $y(n)$, and $e(n)=x(n)-y_{w}(n)$ is the error signal where the adaptive filter output signal is computed as:

$$
y_{w}(n)=\boldsymbol{w}^{T}(n) \boldsymbol{y}(n) .
$$

Ideally, $W(z)$ is expected to generate a replica of $y_{f}(n)$, so that $u(n)=e(n) \approx s(n)$. However, the input and the desired-response signals of $W(z), y(n)$ and $x(n)$, respectively, are correlated with each other and would result in a biased convergence [10]. A simple approach to perform decorrelation is to use an appropriate delay either in the cancelation path [1], or in the forward path [11]; however, it degrades the speech quality. Another solution is to filter the error and/or input signal of $W(z)$, through appropriate 
decorrelation filters, before being used in the update equation of the NLMS algorithm [12], resulting in the so-called Filtered-x adaptive algorithm [13]. In [14], a prediction error method (PEM)-based adaptive feedback canceller has been proposed. The main assumption here is that the desired signal is an autoregressive (AR) process generated from a white noise. Unfortunately, this assumption is not satisfied for periodic signals. A filter bank-based frequency-domain technique has been investigated for AFC [15]. The frequency-domain techniques, however, result in an increased computational load and require a lot of battery power [6]. For time-domain continuous AFC, a dual microphone-based solution has been proposed where two microphones are used to pick the input signal and dual adaptive filters are employed to perform AFC [16, 17]. Those techniques have obvious physical and computational limitations.

Another solution is a noncontinuous adaptation, or an open-loop algorithm in which the hearing aid forward path is broken and a probe signal is injected during particular intervals, for example, when howling is detected by an appropriate oscillation detector [18]. The ON/OFF switching of the probe signal produces annoying effects to the hearing aid user. A continuous injection of probe noise has been considered, however, either the level of the probe noise must be kept low to have an appreciable signal-to-noise ratio (SNR) [19], or an appropriate masking filter be introduced to perceptually mask the probe signal [20].

Recently we have investigated a two adaptive filters-based method in [22] which is based on preliminary results reported in [21]. In [22], essentially: 1) we proposed a structure comprising two adaptive filters $W_{1}(z)$ and $W_{2}(z)$, where $W_{1}(z)$ is the same as in the conventional approach (Fig. 2), and $W_{2}(z)$ is excited by a probe signal, 2) an appropriate delay is inserted in the forward path of the hearing aid, and hence delay-based adaptive filtering [23] is used to adapt the two adaptive filters, 3) an efficient strategy is developed to transfer the weights between the two adaptive filters such that both adaptive filters give a good estimate of $F(z), 4)$ the problem of biased convergence is mitigated by freezing the adaptation once a good solution is obtained, and finally, 5) a time-varying gain is proposed to control the level of added probe noise: a large value is used at the start-up for a fast convergence, and probe signal gain is reduced to a small value as the system converges thus achieving appreciable SNR at the steady-state.

\section{Entrainment}

In order to understand the basic concept of entrainment, we quote from [24]: THE term 'entrainment' refers to the process by which independent rhythmical systems interact with each other. 'Independent rhythmical systems' can be of many types: what they have in common is some form of oscillatory activity (usually periodic or quasi-periodic in nature); they must be independent in the sense of 'selfsustaining', i.e. able to be sustained whether or not they are entrained to other rbythmical systems (thus sympathetic vibration, as when a violin's soundboard vibrates at the same frequency as one of its strings, is not an example of entrainment). In order for interaction to take place some form of coupling must exist between the rhythmical systems, and this too can take many forms. This process of interaction may result in those systems synchronising, in the most common sense of aligning in both phase and period, but in fact entrainment can lead to a wide variety of behaviours.

The adaptive feedback canceller, using NLMS algorithm for example, exploits correlation between the received (speaker) signal and the acoustic feedback signal. When the hearing aid equipped with adaptive filter is stimulated with a periodic/tonal signal from the environment, now there are two independent oscillatory or strongly auto-correlated phenomenon present in the hearing aid. The AFC system (trying) to model and neutralize oscillations caused by acoustic feedback, and the oscillatory or periodic nature of the excitation signal with strong auto-correlation characteristics. The adaptive filter cannot differentiate this correlation from that due to the acoustic feedback, and borrows characteristics of the periodic signal in trying to trace this as well as an undesired correlation. This would result in a non-optimal feedback cancellation, and produces entrainment artifacts [25], [26]. The entrainment causing periodic input signal and the affected feedback canceller filter are called the entraining signal and the entrained filter, respectively. The entrainment artifacts include whistle-like sounds that may or may not be harmonically related to the input tonal signal. These artifacts are very annoying to the 


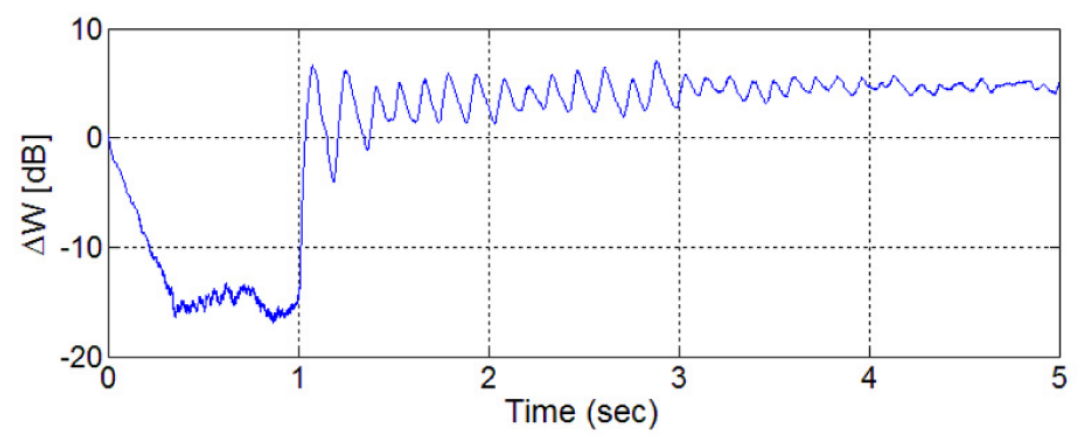

Fig. 2. The time evolution of misalignment (4) in AFC filter $W(z)$ of NLMS-based conventional AFC shown in Fig. 1(b).

users, and furthermore, can result in a reduced output signal quality [27].

As an illustration of entrainment in hearing aids, a typical simulation is performed for hearing aid equipped with NLMS-algorithm based AFC (as shown in Fig. 1(b)). The feedback path $F(z)$ is adopted from [28], and is modeled as an FIR filter with tap-weight length $M=32$. The AFC filter $W(z)$ is also an FIR filter of tap-weight length $M=32$, and is initialized by a null vector. The sampling frequency is $F_{s}=8 \mathrm{kHz}$. The step-size and regularization parameters in Eq. (2) are selected as $\mu=2.5 \times 10^{-2}$ and $\delta=1 \times 10^{-4}$, respectively. The hearing aid processing unit is modeled as a gain-delay system with the transfer function being given as $G(z)=K z^{-\Delta}$, where gain and delay are selected as $K=5$ and $\Delta=10$ (samples), respectively. A five second signal is generated which comprises a white noise for the first 1 sec, and a tonal signal of $1350 \mathrm{~Hz}$ for the rest of the time. The amplitude of tonal signal is adjusted to have almost similar level for the overall signal. The objective for initial white noise part is to get a good estimate of the acoustic feedback path before the onset of tonal signal. The performance of AFC can be judged on the basis of normalized misalignment being computed as:

$$
\Delta W[\mathrm{~dB}]=10 \log \left\{\frac{\|\boldsymbol{f}(n)-\boldsymbol{w}(n)\|^{2}}{\|\boldsymbol{f}(n)\|^{2}}\right\},
$$

where $\|\cdot\|$ denotes Euclidean norm. Fig. 2 shows time evolution of $\Delta W[\mathrm{~dB}]$, where we observe that the misalignment decreases to a low value till the white noise is present. After the onset of tonal signal at $1 \mathrm{sec}$, the AFC filter $W(z)$ diverges to a non-optimal solution. Further, we observe a lot of oscillations in the misalignment curve. Fig. 3 shows time-series and spectrogram plots of the received (speaker) output signal $y(n)$ in comparison with the input signal $s(n)$. During the first $1 \mathrm{sec}$, the output signal is spectrally broad with roughly constant amplitude, and thus closely resembles the input white noise signal. However, following the onset of $1350 \mathrm{~Hz}$ tonal signal at $1 \mathrm{sec}$, we observe significant changes in the output signal. As shown in Fig. 3(b): 1) there is a noticeable reduction in the output level soon after tonal signal starts at $1 \mathrm{sec}$, and 2) rapidly fluctuating strong bursts of new frequencies have been generated which are harmonically unrelated with the input signal. It is worth mentioning that entrainment introduces chaotic-like artifacts, and above is just a typical example. We may observe different behaviors by changing, for example, the tap-weight length of acoustic feedback path $F(z)$ and/or AFC filter $W(z)$, the sampling frequency, or the frequency of input tonal signal. Below is the non-exhaustive summary of entrainment effects (see [26] for details):

1. Entrainment degrades the estimate of the feedback signal, because its response gets distracted to the auto-correlated signal input. Therefore, the system has decreased feedback cancellation.

2. Entrainment causes attenuation of the input stimulus signal. 


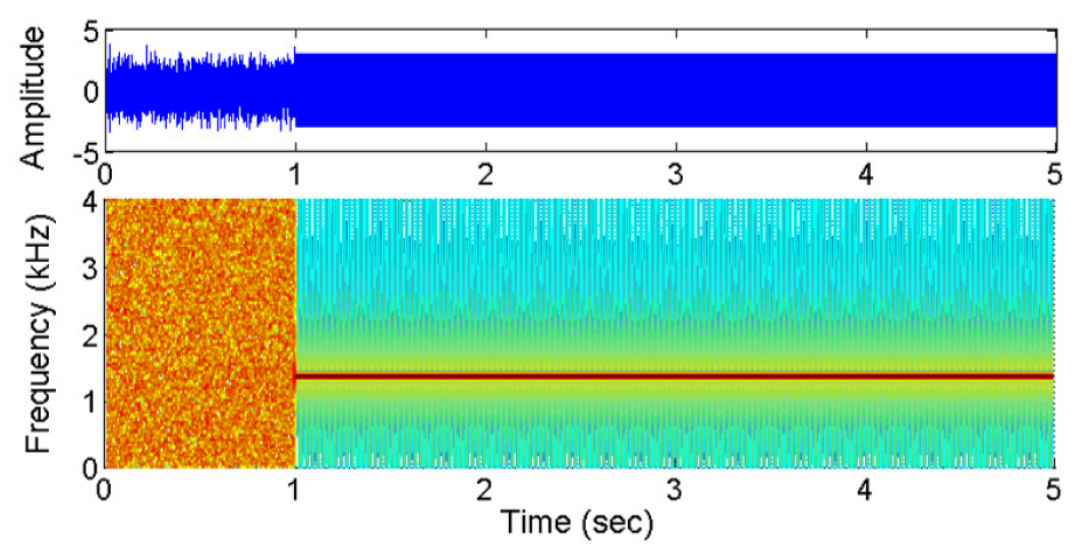

(a)
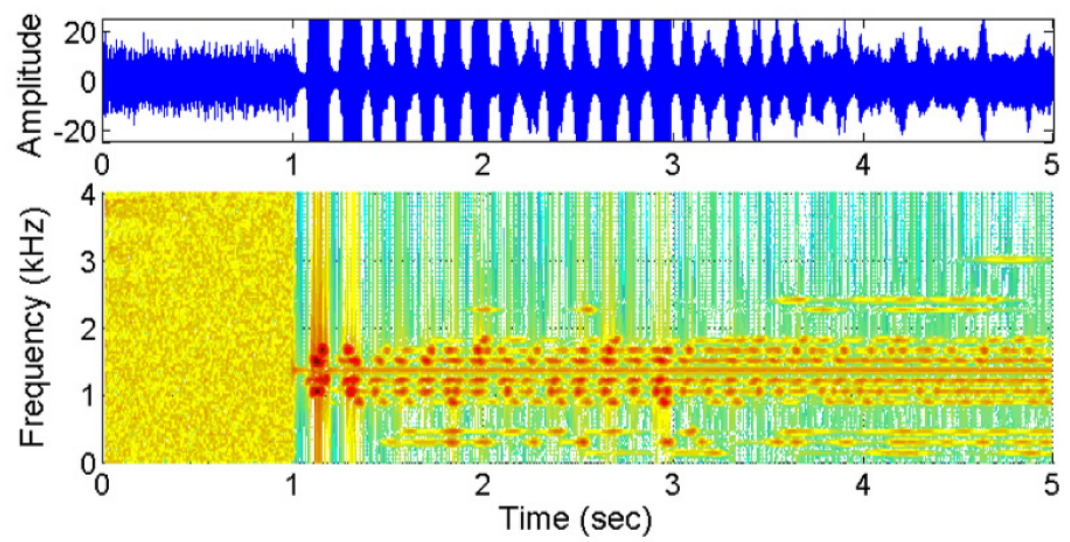

(b)

Fig. 3. The time-series (top) and corresponding spectrogram (bottom) plots for (a) the input signal $s(n)$, and (b) the received (speaker) output signal $y(n)$, in NLMS-based conventional AFC shown in Fig. 1(b).

3. Entrainment increases instability of the system. Once the periodic input stimulus is removed, the entrained system might immediately act as a feedback generator itself, which can cause sustained oscillation.

4. Entrainment severely affects the smaller coefficients at the tail of filter, as these coefficients are more likely to be mistuned by the entraining input signal.

Thus avoiding occurance of entrianment is intimately related to the efficient feedback cancellation performance of hearing aids. Upto the best knowledge of Authors, the most comprehensive attempt to understand and reduce entrainment in hearing aids can be attributed to the $\mathrm{PhD}$ thesis [29]. This work has resulted in a number of US patents including [25, 30, 31, 32]. An interesting argument is hypothesized in [30]: The entrainment can be avoided by analyzing the denominator polynomial [see in Eq. (4) below] for stability, and changing the adaptation rate of the system depending on the position of poles. The closed loop transfer function is being given as:

$$
H(z)=\frac{G(z)}{1-G(z)\left(F_{0}(z)-W(z)\right)} .
$$

where $F_{0}(z)$ denotes the initial (good) estimate of acoustic feedback path $F(z)$ obtained by offline measurements being carried out with white noise at the start-up of hearing aid. A few results have been 
presented in Fig. 3 and Fig. 4 in [30], which show that pole stabilization does improve hearing aid effectiveness in avoiding entrainment.

In [27], it has been observed (via many simulations) that entrained filter 'looks' more like the input signal, whereas a 'good' feedback canceller filter accurately cancelling the feedback signal does not carry any characteristics from the input sound signal. Thus correlation between the filter coefficients and the input signal can be used to see if the filter has been entrained. Once filter enters entrainment, it can be replaced with 'good' estimate. The proposal in [32] employs AR predictor to predict the existence of correlated periodic input. If such input is detected, then the step-size of feedback canceller filter is reduced to slow down the convergence of feedback canceller to avoid the entrainment. The question "What if feedback path changes during the occurrence of periodic/tonal input signal?" remains unanswered.

Recently, Strasser and Puder have attempted to improve efficiency of the acoustic feedback cancellation in hearing aids [33,34]. Noting 'The standard adaptive feedback cancellation algorithms suffer from a biased adaptation if the input signal is spectrally colored or tonal, as it is for speech and music signals. Due to this bias distortion artifacts (entrainment) are generated.', their proposal is to use appropriate sub-band filtering to distinguish between correlation resulting from the input signal and from feedback path changes. These correlation detection are used to increase the performance of the feedback cancellation system. This avoids occurrence of entrainment artifacts.

\section{Concluding Remarks}

In the light of discussion presented in [24], entrainment can be considered as an inherent characteristics of the hearing aid equipped with adaptive feedback canceller, when excited by periodic/tonal-like signal. Once hearing aid becomes entrained, then it is very difficult to recover the stability even if the periodic signal has been removed [26]. Though there have been some research in developing methods to quantify and avoid entrainment [25]-[32]; we believe that entrainment results in a chaotic-like artifact, and some further research is needed to develop measure to quantify its behavior. The signal processing research community has been very active for acoustic feedback and howling cancellation. These efforts need to be continued such that we have AFC being very efficient in canceling the howling, i.e. one oscillation is already been taken care of. Thus periodic or tonal input will not have any other oscillatory process available for entrainment to occur. Indeed, this is the basic idea behind recent works [22], [33], and [34].

\section{References}

[1] D. K. Bustamante, T. L. Worrall, and M. J. Williamson, "Measurement and adaptive suppression of acoustic feedback in hearing aids," in Proc. IEEE ICASSP 1989, pp. 2017-2020.

[2] J. Maxwell and P. Zurek, "Reducing acoustic feedback in hearing aids," IEEE Trans. Speech Audio Process., vol. 4, pp. 304-313, 1995.

[3] B. W. Edwards, "Signal processing techniques for a DSP hearing aid," in Proc. IEEE ISCAS 1998, vol. VI, pp. 586-589.

[4] A. Kaelin, A. Lindgren, and S. Wyrsch, "A digital frequency domain implementation of a very high gain hearing aid with compensation for recruitment of loudness and acoustic echo cancellation," Signal Process., vol. 64, pp. 71-85, 1998.

[5] J. M. Kates, "Constrained adaptation for feedback cancellation in hearing aids," J. Acoust. Soc. Am., vol. 106, pp. 1010-1019, 1999.

[6] J. M. Kates, Digital Hearing Aids. Plural Publishing, 2008.

[7] T. van Waterschoot and M. Moonen, "Fifty years of acoustic feedback control: state of the art and future challenges," Proc. IEEE, vol. 99, no. 2, pp. 288-327, Feb. 2011. 
[8] A Spriet, M. Moonen, and J. Wouters, "Evaluation of feedback reduction techniques in hearing aids based on physical performance measures," J. Acoust. Soc. Am., vol. 128, no. 3, pp. 1245-1261, Sept. 2010.

[9] S. C. Douglas, "A family of normalized LMS algorithms," IEEE Signal Process. Lett., vol. 1, no. 3, pp. 49-51, 1994.

[10] M. G. Siqueira, and A. Alwan, "Steady-State analysis of continuous adaptation in acoustic feedback reduction systems for hearing-aids," IEEE Trans. Speech Audio Process., vol. 8, no. 4, pp. 443-453, 2000 .

[11] P. Estermann and A. Kaelin, "Feedback cancellation in hearing aids: Results from using frequencydomain adaptive filters," in Proc.IEEE ISCAS 1994, pp. 257-260.

[12] J. Hellgren, "Analysis of feedback cancellation in hearing aids with filtered-x LMS and the direct method of closed loop identification," IEEE Trans. Speech Audio Process., vol. 10, no. 2, pp. 119131, 2002.

[13] H. Sakai and H. Fukuzono, "Analysis of adaptive filters in feedback cancellation for sinusoidal signals," in Proc. APSIPA-ASC 2009, pp. 430-433.

[14] A. Spriet, I. Proudler, M. Moonen, and J. Wouters, "Adaptive feedback cancellation in hearing aids with linear prediction of the desired signal," IEEE Trans. Signal Process., vol. 53, no. 10, pp. 3749--3763, 2005.

[15] R. Vicen-Bueno, A. Martínez-Leira, R. Gil-Pita, and M. Rosa-Zurera, "Modified LMS-based feedback-reduction subsystems in digital hearing aids based on WOLA filter bank," IEEE Trans. Instrum. Meas., vol. 58, no. 9, pp. 3177-3190, 2009.

[16] C. R. C. Nakagawa, S. Nordholm, and W. Y. Yan, "Dual microphone solution for acoustic feedback cancellation for assisitve learning," in Proc. IEEE ICASSP 2012, pp. 149-152.

[17] C. R. C. Nakagawa, S. Nordholm, and W. Y. Yan, "Analysis of two microphone method for feedback cancellation,” IEEE Signal Process. Lett., vol. 22, no. 1, pp. 35-39, 2015.

[18] J. E. Greenberg, P. M. Zurek, and M. Brantley, "Evaluation of feedback-reduction algorithms for hearing aids," J. Acoust. Soc. Am., vol. 108, no. 5, pp.2366-2376, 2000.

[19] M. Guo, S. H. Jensen, and J. Jensen, "Novel acoustic feedback cancellation approaches in hearing aid applications using probe noise and probe noise enhancement," IEEE Trans. Audio Speech Lang. Process., vol. 20, no. 9, pp. 2549-2563, 2012.

[20] C. R. C. Nakagawa, S. Nordholm, and W. Y. Yan, "Feedback cancellation with probe shaping compensation,” IEEE Signal Process. Lett., vol. 21, no. 3, pp. 365-369, 2014.

[21] M. T. Akhtar, and A. Nishihara, "Acoustic feedback neutralization in digital hearing aids - A two adaptive filters-based solution," in Proc. IEEE ISCAS 2013, May 19-23, 2013, Beijing, China, pp. 529-532.

[22] M. T. Akhtar, and A. Nishihara, "Automatic tuning of probe noise for continuous acoustic feedback cancelation in hearing aids," in Proc. EUSIPCO 2016, August 29 - September 2, 2016, Budapest Hungary, pp. 888-892.

[23] A. Mader, H. Puder, and G. U. Schmidt, "Step-size control for acoustic echo cancellation filters-An overview,” Signal Process., vol. 4, pp. 1697-1719, 2000.

[24] M. Clayton, "What is Entrainment? Definition and applications in musical research," Empirical Musicology Revierw, vol. 7, no. 1-2, 2012.

[25] L. Theverapperuma, "Entrainment avoidance with a transform domain algorithm," US Patent, 8,509,465 B2, Aug. 13, 2013.

[26] A. Salvetti, H. P. Natarajan, and J. S. Kindred, "Output phase modulation entrainment containment for digital filters," US Patent, 2014/0098967A1, Apr. 10, 2014.

[27] H. P. Natarajan, and J. S. Kindred, "Method and apparatus to reduce entrainment-related artifacts for hearing assistance systems," US Patent, 2011/0116667A1, May 19, 2011. 
[28] K. A. Lee, W. S. Gan, and S. M. Kuo, Subband Adaptive Filtering: Theory and Implementation. Wiley, 2009.

[29] L. S. Theverapperuma, "Entrainment: Adaptive feedback cancelling electroacoustic system dynmaics," Ph.D. thesis, University of Minnesota, 2007. Available: http://gradworks.umi.com/32/68/3268352.html

[30] L. Theverapperuma, "Entrainment avoidance with pole stabilization," US Patent, 2008/0095389, Apr. 24, 2008.

[31] L. Theverapperuma, "Entrainment avoidance with a gradient adaptive lattice filter," US Patent, 8,452,034 B2, May 28, 2013.

[32] L. Theverapperuma, H. P. Natarajan, A. Salvetti, and J. S. Kindred, "Entrainment avoidance with an auto regressive filter," US Patent, 8,681,999 B2, Mar. 25, 2014.

[33] F. Strasser and H. Puder, "Adaptive feedback cancellation for realistic hearing aid applications," IEEE/ACM T. Audio Speech Lang. Process., vol. 23, no. 12, pp. 2322-2333, Dec. 2015.

[34] F. Strasser and H. Puder, "Correlation detection for adaptive feedback cancellation in hearing aids," IEEE Sig. Precess. Lett., vol. 23, no. 7, pp. 979-983, Jul. 2016. 